\title{
Scanning SQUID Microscopy Observation of Grain Boundary Junction in Tri-Phase Epitaxy $\mathrm{NdBa}_{2} \mathrm{Cu}_{3} \mathrm{O}_{7-\delta}$ Thin Film
}

\author{
Shunichi Arisawa, Kyungsung Yun*, Kazuya Mochiduki, Ienari Iguchi, Takeshi Hatano, \\ Huabing Wang, and Akira Ishii \\ National Institute for Materials Science, 1-2-1 Sengen, Tsukuba, Ibaraki 305-0047, Japan \\ Fax: 81-29-859-2801, e-mail: arisawa.shunichi@nims.go.jp \\ *Present Affiliation: Sony Chemical \& Information Device Corporation, Tochigi, Japan
}

\begin{abstract}
High quality grain boundary junction of $\mathrm{NdBa}_{2} \mathrm{Cu}_{3} \mathrm{O}_{7-\delta}$ (NBCO) thin films was fabricated by Tri-Phase Epitaxy technique. Current flows in the film with the junction and artificial defects were investigated by Scanning SQUID microscopy. Magnetic signals generated by the current in the sample were clearly observed. Quantized magnetic vortices close to the grain boundary were also studied.
\end{abstract}

Key words: superconductor, scanning probe microscopy, thin film, grain boundary

\section{INTRODUCTION}

These days, much attention has been paid to Josephson junction and magnetic flux quantum (vortex) based devices such as single flux quantum devices, qubit, SQUID (Superconducting Quantum Interference Device), intrinsic Josephson junction terahertz devices. Especially, fluxonics devices using high Tc oxide superconductors are quite promising since they have unique characteristics other than high transition temperature e.g. $d$-wave asymmetry, large anisotropy, and self-organized intrinsic Josephson junction properties.

Scanning SQUID Microscopy (SSM) is a powerful tool to reveal the nature of vortices in intrinsic Josephson junction and other conventional Josephson junctions. Lorentz force induced by a Current flow in a sample drives and moves vortices in superconductors [1]. Recently, we have directly visualized the motion of interlayer vortices, so-called Josephson vortices, in $\mathrm{La}_{1.87} \mathrm{Sr}_{0.13} \mathrm{CuO}_{4}$ single crystal driven by Lorentz force by using SSM [2].

For designing Josephson junction based devices, behavior of small current flow in thin films is quite important for practical applications since the vortices and currents interact in a sample. In addition to the reason, defects in the sample can be characterized by the imaging the disturbed current flow. Use of SSM is not limited to vortex physics mentioned above but it can be applied to evaluate the current flows in the circuits by observing the magnetic field generated by the currents in the film.

On the other hand, one of the most critical problems to develop electronic devices based on oxide thin films is defects in crystals. Defects in films work unwanted weak links and pinning centers. Tri-Phase epitaxy (TPE) technique developed by Yun et al. is a promising method to overcome this problem [3]. By this method, high quality single crystalline oxide thin films can be fabricated.

In this paper, we report on the observation of magnetic signals generated by the current flows current flow in oxide superconducting thin films by SSM under very weak magnetic field region. A high quality grain boundary junction based on single crystalline NBCO film fabricated by TPE method was used as a sample. Further, vortices around the grain boundary were studied.

\section{EXPERIMENTAL}

NBCO thin films were fabricated by TPE technique on a $\mathrm{SrTiO}_{3}$ bi-crystal substrate. By using TPE method, single crystalline NBCO thin film can be grown. The sample size was $\sim 10 \mathrm{~mm} \times 10 \mathrm{~mm}$. On the boundary of bi-crystal substrate, artificial grain boundary of NBCO is fabricated and this boundary works as a grain boundary Josephson junction [4]. For current observation of Josephson junction, unwanted grain boundaries are harmful. TPE method can eliminate them. Artificial groove and holes were fabricated in the sample to see the effect of the defects and obstacles.

SQM-2000, an SSM developed by SII Nanotechnology Inc., was used for the measurements [5]. The apparatus utilized $\mathrm{Nb}$ DC SQUID and had a spatial resolution limited by the pickup loop of $\varphi 10 \mu \mathrm{m}$. Both the SQUID and the pickup loop were mounted on a Si chip which was set on a cantilever. The sample was scanned without touching the pickup loop. The detector measures the magnetic flux through the pickup loop virtually normal to the sample surface. The SQUID detects the total magnetic flux through the pickup loop. A permalloy magnetic shield reduced the background magnetic field and a small magnetic field could be generated by a coil wound around the sample holder. The SQUID and the sample were cooled by the continuous flow of pumped liquid He.

Small external field normal to the film surface was applied at room temperature and the sample was then field-cooled to $\sim 3 \mathrm{~K}$ at which the measurements were carried out. 


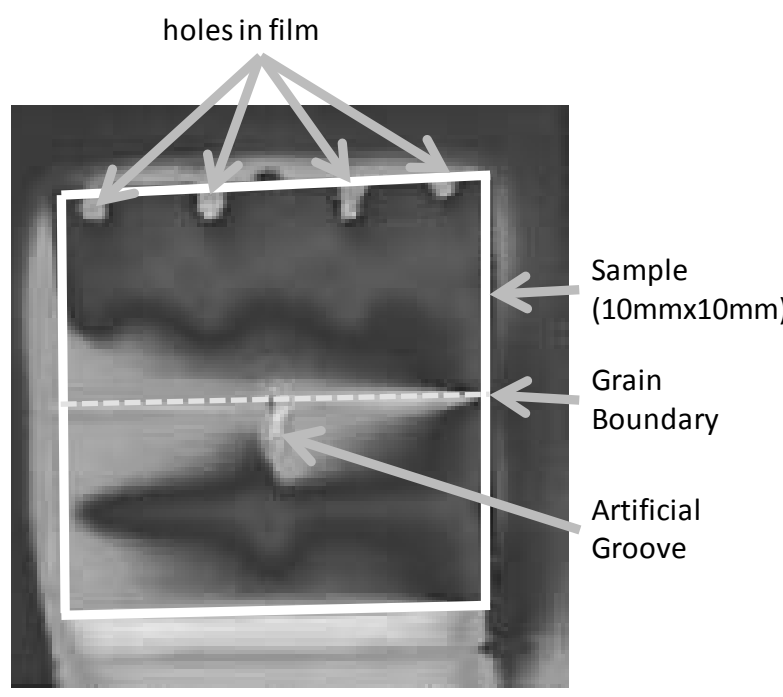

Figure 1. Macroscopic magnetic signal generated by the flow of shielding current was imaged by SSM. Cooled under $3 \mu \mathrm{T}$, Measured under $10 \mu \mathrm{T}$. The artificial groove and holes were also visualized.

\section{RESULTS AND DISCUSSIONS}

Figure 1 shows the macroscopic image of the magnetic signals of the whole sample at $\sim 3 \mathrm{~K}$. The sample was cooled down under $3 \mu \mathrm{T}$ and measured under $10 \mu \mathrm{T}$. The increase of the field after the cooling down causes the shielding current flow in the film. Magnetic signals by the current flow were clearly observed. Reversal of the external magnetic field resulted in the reversal of the sign of the magnetic signal and the response to the switching of the polarity change was reversible. The grain boundary was also clearly observed. The artificial groove and holes were also visualized.

The conversion method from magnetic signal to current flow stream lines is established [6][7] and detailed analyses of our measurements will be published elsewhere.

The grain boundary and quantized vortices close to the line were observed as shown in Fig. 2. The sample was cooled down and measured under $0.5 \mu \mathrm{T}$. A sharp magnetic signal line was observed round the grain boundary. Since the magnetic field was weak, the vortices formed no lattice. The patterns of the vortices did not change even if the external field was increased up to $10 \mu \mathrm{T}$. The vortices were pinned even in the TPE single crystalline thin films, which had very little defect in the crystal. Penetration of the magnetic flux into the grain boundary in TPE film was clearly observed as a line. The magnetic field during the cooling process and that during measurement were the same. In contrast to the condition of Fig. 1 , the shielding current was not considered to be flown. The observed signal on the grain boundary is to be attributed to the penetration of the field into the weak-linked junction.

\section{SUMMARY}

We have characterized a high quality NBCO grain

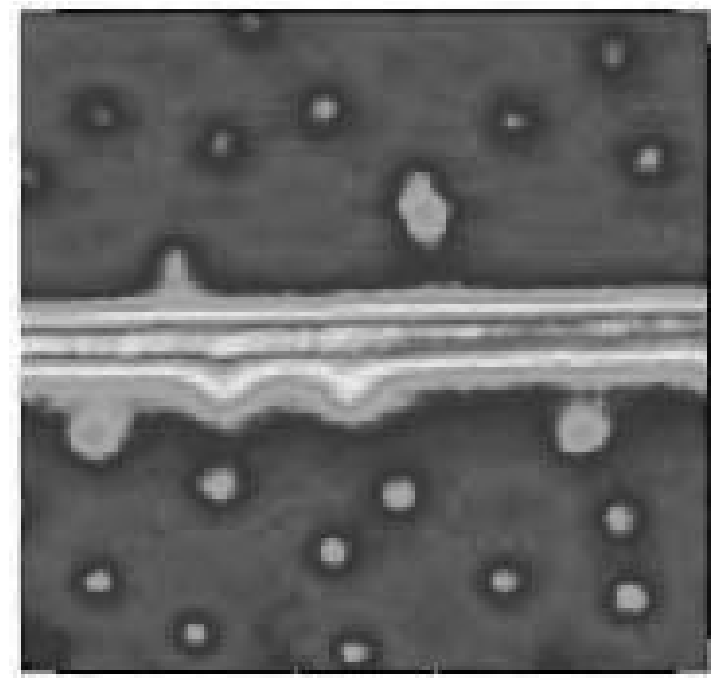

Figure 2. SSM image close to the grain boundary. Scan size was $300 \mu \mathrm{m} \times 300 \mu \mathrm{m}$. $0.5 \mu \mathrm{T}$ field cooled and measured at $0.5 \mu \mathrm{T}$ around $\sim 3 \mathrm{~K}$. The small dots are magnetic flux quantum which carries $\Phi_{0}$. The horizontal line is a grain boundary.

boundary junction fabricated by TPE technique. Macroscopic behaviors of the shielding currents in the $\mathrm{NBCO}$ thin film with grain boundary junction were observed. Quantized vortices close to the grain boundary and the flux penetrating into the grain boundary were also observed.

\section{ACKNOWLEDGEMENT}

Part of this work was supported by JSPS Kakenhi 21560344

\section{REFERENCES}

[1] P. W. Anderson and Y. B. Kim, Rev. Mod. Phys. 36(1964)39.

[2] I. Iguchi, S. Arisawa, K. S. Yun, T. Hatano T. Uchiyama, I. Tanaka, Appl. Phys. Lett. 91, 202511-1 (2007).

[3] K. S. Yun, B. D. Choi, Y. Matsumoto, J. H. Song, N. Kanda, T. Itoh, M. Kawasaki, T. Chikyow, P. Ahmet, and H. Koinuma, Appl. Phys. Lett. 80, 61 (2002).

[4] D. Dimos, P. Chaudhari, J. Mannhart, F.K. Legoues. Phys. Rev. Lett. 61, 219(1988).

[5] T. Morooka, S. Nakayama, A. Odawara and K. Chinone, Jpn. J. Appl. Phys. 38, L119 (1999).

[6] B. J. Roth, N. G. Sepulveda, and J. P. Wikswo, J. Appl. Phys., 65, 361(1988).

[7] K. Abiru, Y. Honda, M. Inoue, T. Kiss, Y. Iijima, K. Kakimoto, T. Saitoh, K. Nakao, Y. Shiohara, Physica $C$ 469, 1450(2009).

(Received February 13, 2010; Accepted February 28, 2010) 